\section{Hypothermia versus Normothermia after Out-of-Hospital Cardiac Arrest}

\author{
J. Dankiewicz, T. Cronberg, G. Lilja, J.C. Jakobsen, H. Levin, S. Ullén, C. Rylander, \\ M.P. Wise, M. Oddo, A. Cariou, J. Bělohlávek, J. Hovdenes, M. Saxena, \\ H. Kirkegaard, P.J. Young, P. Pelosi, C. Storm, F.S. Taccone, M. Joannidis, \\ C. Callaway, G.M. Eastwood, M.P.G. Morgan, P. Nordberg, D. Erlinge, A.D. Nichol, \\ M.S. Chew, J. Hollenberg, M. Thomas, J. Bewley, K. Sweet, A.M. Grejs, \\ S. Christensen, M. Haenggi, A. Levis, A. Lundin, J. Düring, S. Schmidbauer, \\ T.R. Keeble, G.V. Karamasis, C. Schrag, E. Faessler, O. Smid, M. Otáhal, \\ M. Maggiorini, P.D. Wendel Garcia, P. Jaubert, J.M. Cole, M. Solar, O. Borgquist, \\ C. Leithner, S. Abed-Maillard, L. Navarra, M. Annborn, J. Undén, I. Brunetti, \\ A. Awad, P. McGuigan, R. Bjørkholt Olsen, T. Cassina, P. Vignon, H. Langeland, \\ T. Lange, H. Friberg, and N. Nielsen, for the TTM2 Trial Investigators*
}

\section{ABSTRACT}

\section{BACKGROUND}

Targeted temperature management is recommended for patients after cardiac arrest, but the supporting evidence is of low certainty.

\section{METHODS}

In an open-label trial with blinded assessment of outcomes, we randomly assigned 1900 adults with coma who had had an out-of-hospital cardiac arrest of presumed cardiac or unknown cause to undergo targeted hypothermia at $33^{\circ} \mathrm{C}$, followed by controlled rewarming, or targeted normothermia with early treatment of fever (body temperature, $\geq 37.8^{\circ} \mathrm{C}$ ). The primary outcome was death from any cause at 6 months. Secondary outcomes included functional outcome at 6 months as assessed with the modified Rankin scale. Prespecified subgroups were defined according to sex, age, initial cardiac rhythm, time to return of spontaneous circulation, and presence or absence of shock on admission. Prespecified adverse events were pneumonia, sepsis, bleeding, arrhythmia resulting in hemodynamic compromise, and skin complications related to the temperature management device.

\section{RESULTS}

A total of 1850 patients were evaluated for the primary outcome. At 6 months, 465 of 925 patients (50\%) in the hypothermia group had died, as compared with 446 of 925 (48\%) in the normothermia group (relative risk with hypothermia, 1.04; 95\% confidence interval [CI], 0.94 to 1.14; $\mathrm{P}=0.37)$. Of the 1747 patients in whom the functional outcome was assessed, 488 of 881 (55\%) in the hypothermia group had moderately severe disability or worse (modified Rankin scale score $\geq 4$ ), as compared with 479 of $866(55 \%)$ in the normothermia group (relative risk with hypothermia, $1.00 ; 95 \%$ CI, 0.92 to 1.09). Outcomes were consistent in the prespecified subgroups. Arrhythmia resulting in hemodynamic compromise was more common in the hypothermia group than in the normothermia group ( $24 \%$ vs. $17 \%, \mathrm{P}<0.001)$. The incidence of other adverse events did not differ significantly between the two groups.

\section{CONCLUSIONS}

In patients with coma after out-of-hospital cardiac arrest, targeted hypothermia did not lead to a lower incidence of death by 6 months than targeted normothermia. (Funded by the Swedish Research Council and others; TTM2 ClinicalTrials.gov number, NCT02908308.)
The authors' full names, academic degrees, and affiliations are listed in the Appendix. Address reprint requests to Dr. Nielsen at the Department of Anesthesiology and Intensive Care, Intensive Care Unit, Helsingborg Hospital, S Vallgatan 5, 251 87, Helsingborg, Sweden, or atniklas.nielsen@med.lu.se.

*A complete list of TTM2 Trial Investigators is provided in the Supplementary Appendix, available at NEJM.org.

N Engl J Med 2021;384:2283-94. DOI: 10.1056/NEJMoa2100591 Copyright (๑) 2021 Massachusetts Medical Society. 
I NTERNATIONAL GUIDELINES RECOMMEND targeted temperature management to prevent hypoxic-ischemic brain damage in patients with coma after cardiac arrest. ${ }^{1,2}$ The evidence to support these recommendations originated in trials involving patients who had been resuscitated from out-of-hospital cardiac arrest of a presumed cardiac cause and shockable initial rhythms. ${ }^{3,4}$ These trials suggested an increased survival and improved neurologic outcome in patients who underwent hypothermia at $33^{\circ} \mathrm{C}$. A recent trial involving patients who had cardiac arrest with nonshockable rhythm showed better neurologic outcomes with targeted hypothermia at $33^{\circ} \mathrm{C}$ than with targeted normothermia at $37^{\circ} \mathrm{C} .^{5}$ Trials comparing the level of targeted temperature management $\left(33^{\circ} \mathrm{C}\right.$ or $\left.36^{\circ} \mathrm{C}\right)$ and the duration of this management ( 24 hours or 48 hours) have not indicated a dose effect. , $^{6,7}$

Although guidelines strongly recommend targeted temperature management with a constant target between $32^{\circ} \mathrm{C}$ and $36^{\circ} \mathrm{C}$, they also state that the overall evidence is of low certainty. A systematic review that included a meta-analysis and trial sequential analysis indicated that the available trials had high risks of bias and random errors. ${ }^{8}$

Fever has been proposed as a risk factor for an unfavorable neurologic outcome after cardiac arrest, although it is unknown whether there is a causal and modifiable relationship. ${ }^{9}$ Accordingly, we conducted the randomized Targeted Hypothermia versus Targeted Normothermia after Out-of-Hospital Cardiac Arrest (TTM2) trial to assess the beneficial and harmful effects of hypothermia as compared with normothermia and early treatment of fever in patients after cardiac arrest. We hypothesized that at 6 months, the incidence of death would be lower in the hypothermia group than in the normothermia group.

\section{METHODS}

\section{TRIAL DESIGN}

The design of this international, investigatorinitiated superiority trial and its statistical analysis plan have been published previously. ${ }^{10,11}$ The protocol (available with the full text of this article at NEJM.org) was approved by the ethics com- mittees in each participating country. Written informed consent was waived, deferred, or obtained from a legal surrogate, depending on the circumstances, and was obtained from each patient who regained mental capacity. An independent data and safety monitoring committee reviewed the data and performed one prespecified, blinded interim analysis. Additional details on the trial design, including investigator responsibilities, are described in the Supplementary Appendix, available at NEJM.org. There was no commercial funding for the trial.

\section{PATIENTS}

We consecutively screened adults $(\geq 18$ years of age) who had been admitted to the hospital after out-of-hospital cardiac arrest of a presumed cardiac or unknown cause, irrespective of the initial rhythm. All the patients were unconscious and not able to obey verbal commands (score of $<4$ on the Full Outline of Unresponsiveness [FOUR] scale, ${ }^{12}$ which ranges from 0 to 4 , with higher scores indicating better motor function) and did not have a verbal response to pain. Eligible patients had more than 20 consecutive minutes of spontaneous circulation after resuscitation. ${ }^{13}$ The main exclusion criteria were an interval from return of spontaneous circulation to screening of more than 180 minutes, unwitnessed cardiac arrest with asystole as the initial rhythm, and limitations in care. Detailed inclusion and exclusion criteria are provided in the Supplementary Appendix.

\section{RANDOMIZATION AND BLINDING}

After eligibility screening, patients were randomly assigned in a 1:1 ratio to undergo hypothermia or normothermia. Randomization was performed with the use of a Web-based system involving permuted blocks of varying sizes and was stratified according to trial site and coenrollment in the Targeted Therapeutic Mild Hypercapnia after Resuscitated Cardiac Arrest (TAME) trial (ClinicalTrials.gov number, NCT03114033).

Health professionals caring for the trial patients were aware of the trial-group assignments because of inherent problems with blinding body temperature. The physicians assessing neurologic prognosis, assessors of functional outcome, and study administrators were unaware of the trial-group assignments. During the analysis 
phase, the statisticians and authors were unaware of the trial-group assignments, which were identified as $\mathrm{Y}$ and $\mathrm{Z}$. A manuscript was written for each scenario before the randomization code was broken. ${ }^{14}$

\section{TRIAL INTERVENTION}

The intervention period of 40 hours began at the time of randomization. Patients who were assigned to undergo hypothermia were immediately cooled with a surface or intravascular temperature-management device to a target temperature of $33^{\circ} \mathrm{C}$. This target was maintained until 28 hours after randomization, followed by rewarming to $37^{\circ} \mathrm{C}$ in hourly increments of one third of a degree. In the normothermia group, the aim was to maintain a temperature of $37.5^{\circ} \mathrm{C}$ or less. If conservative and pharmacologic measures were insufficient and the body temperature reached $37.8^{\circ} \mathrm{C}$ or higher, cooling with a surface or intravascular temperature-management device was initiated with a target temperature of $37.5^{\circ} \mathrm{C}$. No active warming or cooling was provided for patients in the normothermia group who had a spontaneous body temperature below $37.8^{\circ} \mathrm{C}$. Sedation was mandated in both groups until the end of the intervention period. After the intervention period, a normothermic target $\left(36.5^{\circ} \mathrm{C}\right.$ to $37.7^{\circ} \mathrm{C}$ ) was maintained until 72 hours after randomization in patients who remained sedated or comatose. Details of the trial interventions are provided in the Supplementary Appendix.

\section{ASSESSMENT OF NEUROLOGIC PROGNOSIS AND WITHDRAWAL OF LIFE SUPPORT}

At 96 hours after randomization or later, a physician who was unaware of the intervention assignments performed a neurologic assessment of patients who remained in the intensive care unit (ICU). The physician assessed whether the criteria for a likely poor neurologic outcome were present.

All decisions about withdrawal of life-sustaining therapy were at the discretion of the treating physician, guided by the protocol. After assessment of neurologic prognosis, withdrawal of life-sustaining therapies due to a presumed poor neurologic prognosis was allowed. (Details regarding the neurologic evaluation are provided in the Supplementary Appendix.)

\section{PRIMARY AND SECONDARY OUTCOMES}

The primary outcome was death from any cause at 6 months. The main secondary outcome was a poor functional outcome at 6 months, defined as a score of 4 to 6 on the modified Rankin scale. ${ }^{15,16}$ Scores on the modified Rankin scale range from 0 to 6 , with 0 representing no symptoms, 1 no clinically significant disability, 2 slight disability, 3 moderate disability, 4 moderately severe disability, 5 severe disability, and 6 death. A trained outcome assessor used a structured questionnaire to evaluate the patient's condition. The functional score was determined after faceto-face or telephone interviews with patients, relatives, and health care providers. ${ }^{17}$

If a structured assessment could not be completed, a binary assessment based on all available data (including medical records) was performed; functional outcome was classified as "good" or "poor" on the basis of a dichotomized modified Rankin scale (a score of 0 to 3 or 4 to 6). This post hoc approach was used because of the restrictions imposed during the coronavirus disease 2019 pandemic.

Other secondary outcomes were the number of days the patient was alive and out of the hospital until day 180, survival determined in a time-to-death analysis, and health-related quality of life, which was assessed with the use of the visual-analogue scale on the European Quality of Life-5-Dimension-5-Level questionnaire (EQ-5D-5L), which ranges from 0 to 100 , with higher scores indicating better health status as assessed by the patient. ${ }^{15}$ Verification of trial data and the outcome measures are described in the Supplementary Appendix.

\section{ADVERSE EVENTS}

Prespecified adverse events were pneumonia, sepsis, bleeding, arrhythmia resulting in hemodynamic compromise, and skin complications related to the device used for targeted temperature management. Definitions of these adverse events are provided in the Supplementary Appendix.

\section{STATISTICAL ANALYSIS}

We estimated that a sample of 1862 patients would provide $90 \%$ power to detect a relative reduction of $15 \%$ in the risk of death in the hypothermia group, as compared with the normo- 
Table 1. Baseline Characteristics of the Intention-to-Treat Population.*

\begin{tabular}{|c|c|c|}
\hline Characteristic & $\begin{array}{l}\text { Hypothermia } \\
(\mathrm{N}=930)\end{array}$ & $\begin{array}{l}\text { Normothermia } \\
\quad(\mathrm{N}=931)\end{array}$ \\
\hline \multicolumn{3}{|l|}{ Demographic characteristics } \\
\hline Age $-y r$ & $64 \pm 13$ & $63 \pm 14$ \\
\hline Male sex — no. (\%) & $742(80)$ & 735 (79) \\
\hline \multicolumn{3}{|l|}{ Medical history } \\
\hline Hypertension — no. (\%) & $345(37)$ & $298(32)$ \\
\hline Diabetes — no. (\%) & $173(19)$ & $167(18)$ \\
\hline Myocardial infarction — no. (\%) & $139(15)$ & $154(17)$ \\
\hline $\mathrm{PCl}-$ no. (\%) & $130(14)$ & $140(15)$ \\
\hline Coronary-artery bypass grafting — no. (\%) & $73(8)$ & $76(8)$ \\
\hline Heart failure - no. (\%) & $90(10)$ & $93(10)$ \\
\hline NYHA III or IV heart failure — no./total no. (\%)† & $20 / 906(2)$ & $23 / 904(3)$ \\
\hline Median Charlson comorbidity index (IQR) $\ddagger$ & $3(2-4)$ & $3(1-4)$ \\
\hline \multicolumn{3}{|l|}{ Characteristics of the cardiac arrest - no. (\%) } \\
\hline \multicolumn{3}{|l|}{ Location at cardiac arrest } \\
\hline Place of residence & $487(52)$ & $491(53)$ \\
\hline Public place & $338(36)$ & $320(34)$ \\
\hline Other & $105(11)$ & $120(13)$ \\
\hline Bystander-witnessed cardiac arrest & $850(91)$ & $852(92)$ \\
\hline Bystander-performed CPR & $759(82)$ & $728(78)$ \\
\hline \multicolumn{3}{|l|}{ First monitored rhythm — no. (\%) } \\
\hline Shockable rhythm & $671(72)$ & $700(75)$ \\
\hline Ventricular fibrillation & $576(62)$ & $585(63)$ \\
\hline Nonperfusing ventricular tachycardia & $31(3)$ & $29(3)$ \\
\hline ROSC after bystander-initiated defibrillation & $24(3)$ & $41(4)$ \\
\hline Unknown rhythm, shock administered & $40(4)$ & $45(5)$ \\
\hline Nonshockable rhythm & $259(28)$ & $231(25)$ \\
\hline Pulseless electrical activity & $117(13)$ & $113(12)$ \\
\hline Asystole & $124(13)$ & $100(11)$ \\
\hline Unknown rhythm, no shock administered & $18(2)$ & $18(2)$ \\
\hline Median time from cardiac arrest to sustained ROSC (IQR) - $\min \sqrt{ }$ & $25(16-40)$ & $25(17-40)$ \\
\hline Median time from cardiac arrest to randomization - $\min (\mathrm{IQR})$ & $136(103-170)$ & $133(99-173)$ \\
\hline \multicolumn{3}{|l|}{ Clinical characteristics on admission } \\
\hline Tympanic temperature $-{ }^{\circ} \mathrm{C} \mathbf{9}$ & $35.3 \pm 1.1$ & $35.4 \pm 1.1$ \\
\hline FOUR motor score $\|$ & 0 & 0 \\
\hline Bilateral corneal reflexes present — no./total no. (\%) & $168 / 511(33)$ & $194 / 537(36)$ \\
\hline Bilateral pupillary reflexes present — no./total no. (\%) & $535 / 761(70)$ & $529 / 776(68)$ \\
\hline Arterial $\mathrm{pH} * *$ & $7.2 \pm 0.2$ & $7.2 \pm 0.2$ \\
\hline Arterial lactate level — mmol/liter & $5.9 \pm 4.4$ & $5.8 \pm 4.2$ \\
\hline Shock-no. (\%)+r & $261(28)$ & $275(30)$ \\
\hline ST-segment elevation myocardial infarction — no./total no. (\%) & $379 / 918(41)$ & $370 / 921(40)$ \\
\hline
\end{tabular}


Table 1. (Continued.)

* Plus-minus values are means \pm SD. CPR denotes cardiopulmonary resuscitation, IQR interquartile range, $\mathrm{PCl}$ percutaneous coronary intervention, and ROSC return of spontaneous circulation.

$\dagger$ New York Heart Association (NYHA) heart failure class was not assessed in 51 patients (24 in the hypothermia group and 27 in the normothermia group) who had a history of heart failure.

$\neq$ On the Charlson comorbidity index, each comorbidity category is weighted from 1 to 6 on the basis of adjusted risk of death or resource use, and the sum of the weights produces the score. A score of 0 indicates an absence of known coexisting conditions, and higher scores indicate higher risks of death and greater resource use.

$\int$ For unwitnessed cardiac arrests, the time to ROSC was calculated from the time of the emergency call.

I Tympanic temperature was assessed in 1559 patients.

Full Outline of Unresponsiveness (FOUR) motor scores range from 0 to 4, with higher scores indicating better motor function. The FOUR motor score was assessed in 1696 patients.

$* *$ Arterial $\mathrm{pH}$ was measured in 1829 patients.

t广 The arterial lactate level was measured in 1781 patients.

Shock at admission was defined as a systolic blood pressure of less than $90 \mathrm{~mm} \mathrm{Hg}$ for more than 30 minutes or end-organ hypoperfusion (cool arms and legs, urine output $<30 \mathrm{ml}$ per hour, and heart rate $<60$ beats per minute).

thermia group, at a two-sided alpha level of 0.05 (absolute risk reduction of 7.5 percentage points). The estimated relative risk was based on results from earlier trials of hypothermia for cardiac arrest. ${ }^{10}$ To allow for loss to follow-up and withdrawn consent, a sample size of 1900 was chosen.

The principal trial analyses were performed in the intention-to-treat population, defined as all randomly assigned patients except those for whom consent was withdrawn. Dichotomous outcomes, including the primary analysis, were assessed with the use of a mixed-effects generalized linear model with a logit link with adjustment for stratification variables and were reported as population-level (marginal) relative risks derived by G-computation. Analysis of survival data was performed with Cox regression. For all regression analyses, we tested for an interaction effect between group assignment and assignment in the TAME trial. We made no assumptions regarding the pattern of missing data, which were handled according to the statistical analysis plan..$^{11,18} \mathrm{~A}$ P value of less than 0.05 was considered to indicate statistical significance for the primary outcome. Secondary outcomes are presented with $95 \%$ confidence intervals and were not adjusted for multiplicity. All analyses were performed with the use of R: A Language and Environment for Statistical Computing. ${ }^{19}$

\section{RESULTS}

\section{PATIENTS}

A total of 1900 patients were enrolled between November 2017 and January 2020. Consent could not be obtained or was withdrawn in 37 patients, and 2 patients underwent randomization twice, resulting in an intention-to-treat population of 1861, of whom 930 were assigned to the hypothermia group and 931 to the normothermia group (Fig. S1 in the Supplementary Appendix). Baseline characteristics are reported in Table 1. Details regarding procedures and administered drugs, assessment of neurologic prognosis, withdrawal of life-sustaining therapy, length of ICU and hospital stay, and data regarding coenrollment in the TAME trial are provided in Tables S1 through S6 and Figures S2 and S3.

\section{TEMPERATURE INTERVENTION}

The temperature curves are shown in Figure 1. In the hypothermia group, the median time from the start of the intervention until a temperature of $34^{\circ} \mathrm{C}$ was reached was 3 hours. In this group, 53 of 930 patients $(6 \%)$ were rewarmed before 40 hours after randomization, as allowed by the protocol, primarily because of cardiovascular instability and arrhythmias (Table S7). A total of 882 of 930 patients (95\%) in the hypothermia group and 428 of 931 patients (46\%) in the normothermia group received cooling with a device. Among patients who received cooling, the types of devices used in each treatment group were similar $(70 \%$ surface and 30\% intravascular in the hypothermia group and 69\% surface and $31 \%$ intravascular in the normothermia group). In the hypothermia group, the reasons for not receiving cooling with a device were intracranial hemorrhage, early death, early awakening, hemodynamic instability, and referral for cardiac surgery, whereas the main reason in the normothermia group was not reaching the threshold 


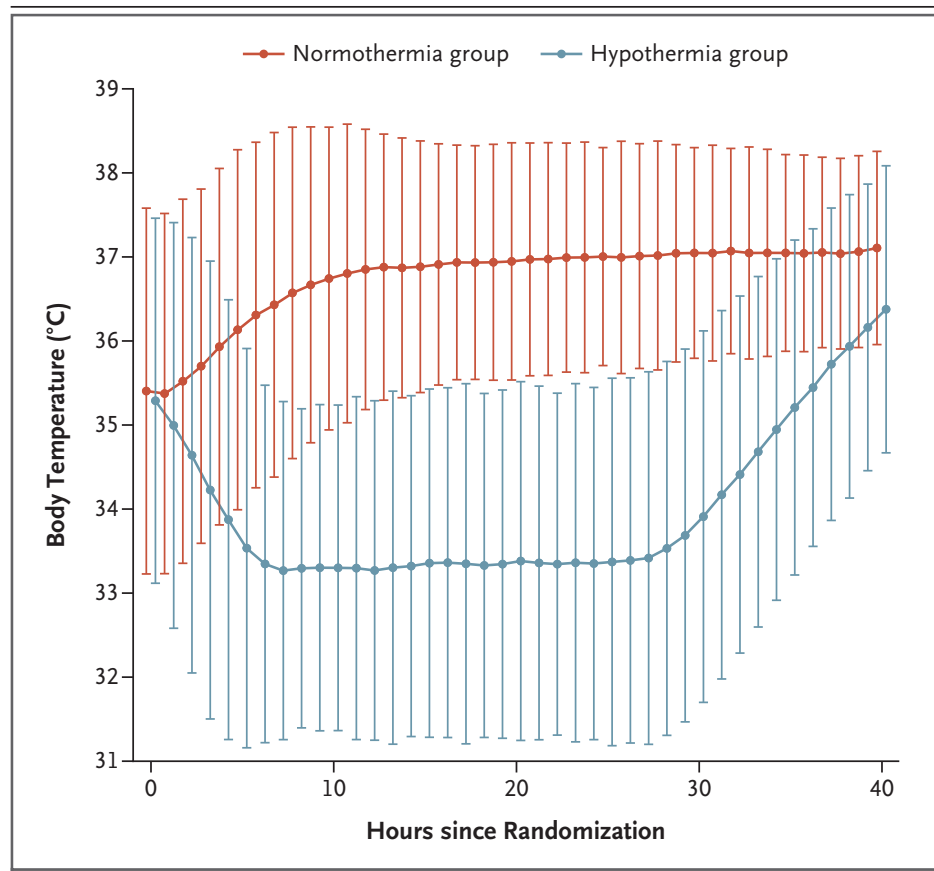

Figure 1. Body Temperature during the Intervention Period.

Shown are body-temperature curves in the hypothermia and normothermia groups for the patients in whom a bladder temperature was recorded. The median number of temperature recordings was 38 in both the hypothermia group and the normothermia group, out of 41 possible recordings. The temperature curves show the means, and the I bars indicate \pm 2 SD $(95 \%$ of the observations are within the error bars). The median time from cardiac arrest to randomization in the trial was 135 minutes. for fever. Additional data regarding temperatures and shivering are available in Figures S4 through S7 and Table S8.

\section{PRIMARY AND SECONDARY OUTCOMES}

Data on the primary outcome were missing for 11 patients (5 in the hypothermia group and 6 in the normothermia group) of 1861 patients overall $(<1 \%)$. At 6 months, 465 of 925 patients (50\%) in the hypothermia group and 446 of 925 patients $(48 \%)$ in the normothermia group had died (relative risk with hypothermia, 1.04; 95\% confidence interval [CI], 0.94 to $1.14 ; \mathrm{P}=0.37$ ). The effect of the temperature intervention on death at 6 months was consistent across the prespecified subgroups (Fig. 2A) and when assessed in a time-to-event analysis (hazard ratio in the hypothermia group, 1.08; 95\% CI, 0.95 to 1.23) (Fig. 3).
Functional outcome was assessed according to the modified Rankin scale in 1747 of 1861 patients (94\%) (Fig. S8). A structured assessment was performed in a face-to-face interview (72\%), by phone $(23 \%)$, or by proxy interview $(5 \%)$. In addition, functional outcome was classified only as "good" or "poor" on the basis of telephone interviews with relatives and health care providers and on the basis of medical records in 37 of 930 patients (4\%) in the hypothermia group and 45 of 931 patients $(5 \%)$ in the normothermia group. In total, functional outcome was assessed in 1829 of 1861 patients (98\%).

At 6 months, 488 of 881 patients (55\%) in the hypothermia group and 479 of 866 patients $(55 \%)$ in the normothermia group had a modified Rankin scale score of 4 to 6 (relative risk with hypothermia, 1.00; 95\% CI, 0.92 to 1.09 ). In the binary assessment of functional outcome, 495 of 918 patients (54\%) in the hypothermia group and 493 of 911 patients (54\%) in the normothermia group had a poor functional outcome (relative risk in the hypothermia group, 1.00; $95 \% \mathrm{CI}, 0.91$ to 1.08$)$. The effect of the temperature intervention on functional outcome was consistent across the prespecified subgroups (Fig. 2B).

Health-related quality of life as assessed with the use of the EQ-5D-5L visual-analogue scale was similar in the hypothermia and normothermia groups, regardless of whether the patients who died were included (with the score on the EQ-5D-5L visual-analogue scale set to 0 ) or only those who survived were assessed (mean betweengroup difference in patients who survived to 6 months, -0.8 points; $95 \% \mathrm{CI},-3.6$ to 2.0 ) (Table S9). The distribution of days when the patients were alive and out of the hospital was similar in the two groups (Fig. S9).

Best-worst and worst-best analyses indicated that missing data did not have the potential to affect the results of the analyses of both death from any cause and functional outcome (Table S10). Additional sensitivity analyses are reported in Table S11. There were no significant interactions between group assignments in the current trial and assignments in the TAME trial for any of the outcomes (range of P for interaction, 0.58 to 0.94 ) (Table S12). 


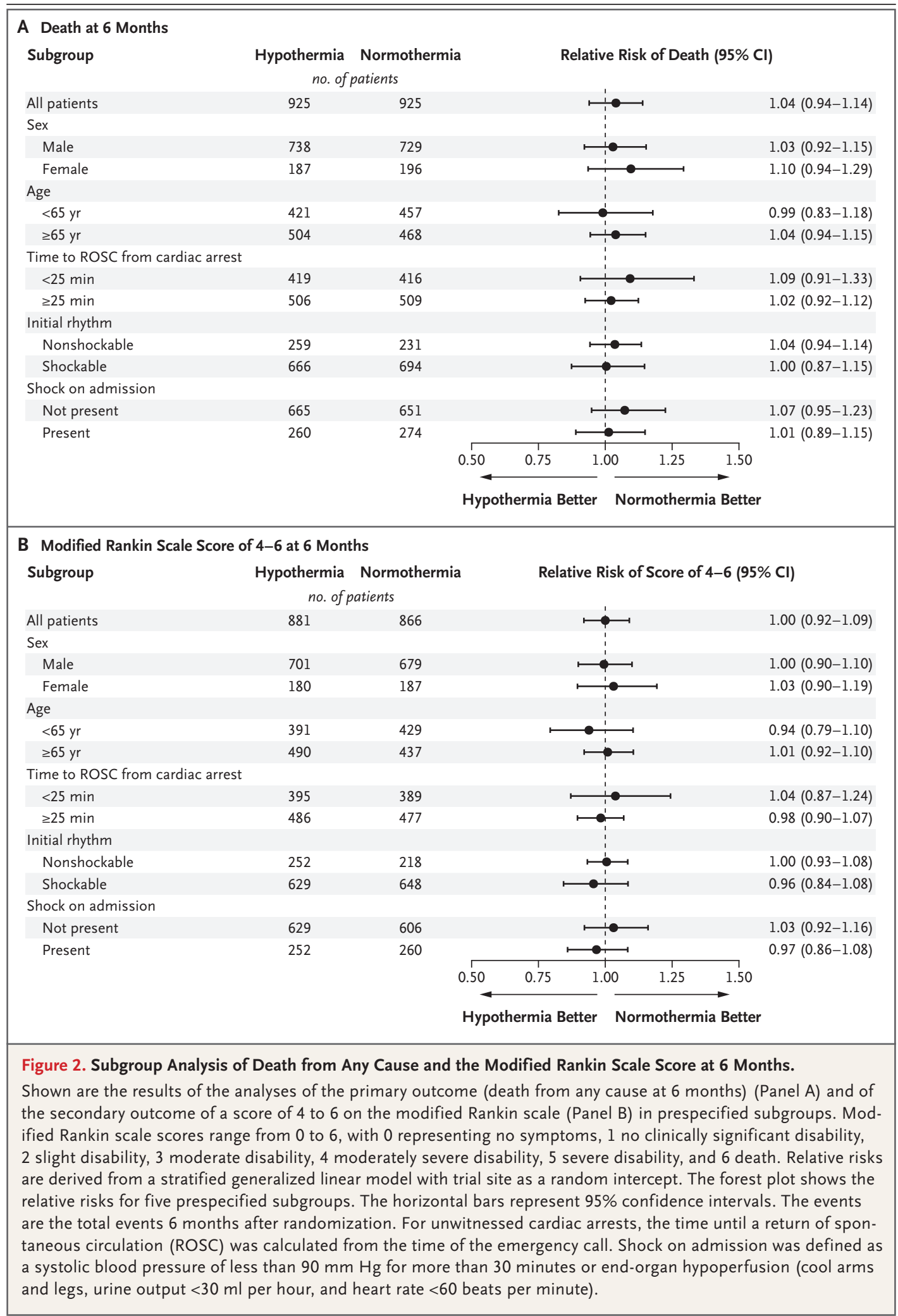

The New England Journal of Medicine 




\section{ADVERSE EVENTS}

Prespecified adverse events are reported in Table 2. Arrhythmias resulting in hemodynamic compromise were more common in the hypothermia group than in the normothermia group (in $24 \%$ vs. $17 \%$; $\mathrm{P}<0.001$ ). There were no significant differences in other prespecified adverse events. Two unexpected serious, possibly intervention-related adverse events occurred in each group: an intravascular device-related thrombosis in one patient in the hypothermia group and two patients in the normothermia group, and bradycardia with worsening hemodynamic function in one patient in the hypothermia group (see the Supplementary Appendix).

\section{DISCUSSION}

In this randomized trial, we compared hypothermia with normothermia in patients with coma who had been resuscitated after out-of-hospital cardiac arrest of a presumed cardiac or unknown cause. There was no significant difference between the two groups with respect to death and poor functional outcome at 6 months. The distribution of scores on the modified Rankin scale between the groups was similar, as was healthrelated quality of life. The results were consistent in the analysis of survival and in prespecified subgroups.

Our results contrast with findings of practice-changing trials published in 2002 in which a benefit of hypothermia was reported. ${ }^{3,4}$ Since then, there have been changes in standards of intensive care that may have influenced intervention effects. ${ }^{20,21}$ Other explanations would be a lower risk of bias in the current trial ${ }^{22}$ and a lower risk of random error with a sample size that was five times the combined enrollment of the earlier trials. ${ }^{23,24}$ Although the patient population we studied differed somewhat from those in previous trials, our subgroup analyses indicate that different eligibility criteria are unlikely to explain the discordance.

Our findings are consistent with those of a recent trial in which hypothermia at $33^{\circ} \mathrm{C}$, as compared with normothermia at $37^{\circ} \mathrm{C}$, in patients with nonshockable rhythms was not shown to reduce mortality. ${ }^{5}$ That trial indicated that hypothermia may improve functional outcomes, but this finding was based on a small number of events and was not replicated in the subgroup of patients with initial nonshockable rhythm in our trial.

The results of the current trial are broadly consistent with the results of our previous TTM (Target Temperature Management $33^{\circ} \mathrm{C}$ versus $36^{\circ} \mathrm{C}$ after Out-of-Hospital Cardiac Arrest) trial. ${ }^{6}$ The combined results of the two trials imply a low likelihood of any meaningful clinical improvement with hypothermia as compared with normothermia, since $36^{\circ} \mathrm{C}$ may be considered to be the lower boundary of normothermia.

It is physiologically plausible that the interval between a cardiac event and the initiation of hypothermia is related to potential benefits of the intervention, a hypothesis that is supported by experiments in animals. ${ }^{25}$ In our trial, patients were cooled at a similar or faster rate than that in most previous trials. ${ }^{3,5-7}$ Since all participating sites in our trial had previous experience with the use of hypothermia, and a large percentage of the patients in our trial underwent randomization at cardiac arrest centers, the cooling rates we observed were probably faster than those that are feasible in current clinical practice. 


\begin{tabular}{|c|c|c|c|c|}
\hline Outcome or Event & $\begin{array}{l}\text { Hypothermia } \\
(\mathrm{N}=930)\end{array}$ & $\begin{array}{l}\text { Normothermia } \\
\quad(N=931)\end{array}$ & $\begin{array}{l}\text { Relative Risk } \\
(95 \% \mathrm{Cl}) *\end{array}$ & P Value \\
\hline $\begin{array}{l}\text { Primary outcome: death from any cause at } 6 \text { mo } \\
\text { - no./total no. (\%) }\end{array}$ & $465 / 925(50)$ & $446 / 925(48)$ & $1.04(0.94-1.14)$ & 0.37 \\
\hline \multicolumn{5}{|l|}{ Main secondary outcome - no./total no. (\%) } \\
\hline $\begin{array}{l}\text { Score of } 4-6 \text { on modified Rankin scale at 6-mo } \\
\text { follow-upt }\end{array}$ & $488 / 881(55)$ & $479 / 866(55)$ & $1.00(0.92-1.09)$ & \\
\hline Poor functional outcome at 6 mo & $495 / 918(54)$ & $493 / 911(54)$ & $1.00(0.91-1.08)$ & \\
\hline \multicolumn{5}{|l|}{$\begin{array}{l}\text { Score on modified Rankin scale at 6-mo follow-up } \\
\text { — no./total no. }(\%) \dagger\end{array}$} \\
\hline 0 & $140 / 881(16)$ & $148 / 866(17)$ & & \\
\hline 1 & $87 / 881(10)$ & $80 / 866(9)$ & & \\
\hline 2 & $132 / 881(15)$ & $127 / 866(15)$ & & \\
\hline 3 & $34 / 881(4)$ & $32 / 866(4)$ & & \\
\hline 4 & $16 / 881(2)$ & $20 / 866(2)$ & & \\
\hline 5 & $7 / 881(1)$ & $13 / 866(2)$ & & \\
\hline 6 & $465 / 881(53)$ & $446 / 866(52)$ & & \\
\hline \multicolumn{5}{|l|}{ Serious adverse events - no./total no. (\%) } \\
\hline $\begin{array}{l}\text { Arrhythmia resulting in hemodynamic com- } \\
\text { promise }\end{array}$ & $222 / 927(24)$ & $152 / 921(16)$ & $1.45(1.21-1.75)$ & $<0.001$ \\
\hline Bleeding & $44 / 927(5)$ & $46 / 922(5)$ & $0.95(0.63-1.42)$ & 0.81 \\
\hline $\begin{array}{l}\text { Skin complication related to device used for } \\
\text { targeted temperature management }\end{array}$ & $10 / 927(1)$ & $5 / 922(<1)$ & $1.99(0.71-6.37)$ & 0.21 \\
\hline Pneumonia & $330 / 927(36)$ & $322 / 921(35)$ & $1.02(0.90-1.15)$ & 0.75 \\
\hline Sepsis & $99 / 926$ (11) & $83 / 922(9)$ & 1.19 (0.90-1.57) & 0.23 \\
\hline
\end{tabular}

Hypothermia did not increase the frequency of pneumonia, sepsis, or bleeding, but arrhythmias causing hemodynamic compromise were more common in the hypothermia group than in the normothermia group. Possible reasons for this include electrolyte disturbances, fluid status, and a temperature effect on cardiac myocytes. ${ }^{26}$

Our trial has several limitations. First, to isolate the effect of hypothermia, both trial groups were treated similarly, except for the temperature intervention. Elements of standard care in the ICU, such as sedation, paralysis, and me- chanical ventilation, were therefore included in the trial protocol in a form that was not necessarily representative of clinical practice. It is unclear what influence these elements had on the outcomes. The trial also included a conservative protocol for assessment of neurologic prognosis and guidance for withdrawal of life support, which may have influenced outcomes. Second, staff members in the ICU were aware of the assigned target temperature during the ICU stay. We aimed to minimize this problem by using outcomes with a low risk of bias, outcome assessors who were unaware of the trial-group 
assignments, and a conservative protocol for determination of the neurologic prognosis and withdrawal of life-supporting therapies. During the analysis and writing process, the investigators, statisticians, and authors were unaware of the temperature-group assignments, and writing of the manuscript was performed in duplicate, with the groups interchanged. Third, since we did not include a control group without temperature management, this trial leaves a knowledge gap regarding whether any temperature management is better than no temperature management. Nonetheless, actual temperatures in the normothermia group were broadly similar to those recorded in the control group of the Hypothermia after Cardiac Arrest trial, in which no temperature management was used. ${ }^{3}$ As compared with that trial, about half the patients in the normothermia group in our trial were cooled with a device. Whether this type of fever control is of benefit must be addressed in a separate trial. Fourth, concomitant care, except for sedation and prognostication, was not part of the protocol and was left to the discretion of participating hospitals. However, sites were instructed to treat the groups similarly, and the stratification for participating hospitals should have balanced intersite differences. Fifth, the trial was limited to out-of-hospital cardiac arrest of a presumed cardiac or unknown cause, so the results are not fully applicable to other presentations of cardiac arrest. However, a lack of cerebral perfusion is the primary cause of hypoxicischemic encephalopathy in cardiac arrest, regardless of where the event occurs or the cause of arrest. Finally, one fifth of the patients were also enrolled in the TAME trial. We did not anticipate any between-trial interaction, an expectation that was supported by our analyses, although such comparisons were probably underpowered.

Our results were consistent across the objective outcome of death from any cause, the clinician-reported functional outcome (as measured on the modified Rankin scale), and patientreported health-related quality of life (as measured on the EQ-5D-5L visual-analogue scale). The large sample size, broad eligibility criteria, and numerous hospitals and countries represented in this trial increase the generalizability.

Patients with coma after out-of-hospital cardiac arrest who were treated with hypothermia did not have a lower incidence of death at 6 months than those who were treated with normothermia.

Supported by independent research grants from nonprofit or governmental agencies (the Swedish Research Council [Vetenskapsrådet], Swedish Heart-Lung Foundation, Stig and Ragna Gorthon Foundation, Knutsson Foundation, Laerdal Foundation, Hans-Gabriel and Alice Trolle-Wachtmeister Foundation for Medical Research, and Regional Research Support in Region Skåne) and by governmental funding of clinical research within the Swedish National Health Service.

Dr. Oddo reports receiving advisory board fees from Neuroptics; Dr. Cariou, receiving lecture fees from Bard Medical; Dr. Saxena, receiving consulting fees from Bard Medical; Dr. Young, receiving lecture fees from Bard Medical; Dr. Storm, receiving lecture fees and travel support from Bard Medical and consulting fees from BrainCool, Pfizer, Sedana Medical, and Xenios; Dr. Taccone, receiving grant support from Bard Medical and ZOLL Medical; Dr. Callaway, holding patent US7269454B2 on methods and devices to guide therapy for ventricular fibrillation with waveform analysis, patent WO2016168181A1 on identification of non-ST elevation ischemic events on electrocardiography, and patent US6438419B1 on a method and apparatus using a scaling exponent for selective defibrillation; Dr. Morgan, receiving fees for coordinating an educational workshop from Bard Medical; Dr. Nichol, receiving grant support, paid to University College Dublin, from AM Pharma and grant support, paid to Monash University, from Baxter Healthcare; Dr. Chew, receiving lecture fees from Edwards Lifesciences; Dr. Levis, receiving donated supplies from Prytime Medical Devices; Dr. Keeble, receiving travel support from Zoll Circulation; Dr. Leithner, receiving lecture fees, paid to Charité Universitätzmedizin, from Bard Medical and ZOLL Medical; Dr. Friberg, receiving fees for academic advising from TEQCool; and Dr. Nielsen, receiving lecture fees from Bard Medical and consulting fees from BrainCool. No other potential conflict of interest relevant to this article was reported.

Disclosure forms provided by the authors are available with the full text of this article at NEJM.org.

A data sharing statement provided by the authors is available with the full text of this article at NEJM.org.

We thank the trial patients, their relatives, and clinical and research staff at all the trial sites; Audrey Shearer and colleagues at Spiral Software; Richard Frobell, Kristina Källén, Ulf Malmqvist, Kathy Rowan, David Harrison, Paul Mouncey, Manu Shankar-Hari, Duncan Young, Karolina Palmér, and Viktor Hultqvist; and the TAME trial group.

\section{APPENDIX}

The authors' full names and academic degrees are as follows: Josef Dankiewicz, M.D., Ph.D., Tobias Cronberg, M.D., Ph.D., Gisela Lilja, O.T., Ph.D., Janus C. Jakobsen, M.D., Ph.D., Helena Levin, M.Sc., Susann Ullén, Ph.D., Christian Rylander, M.D., Ph.D., Matt P. Wise, M.B., B.Ch., D.Phil., Mauro Oddo, M.D., Alain Cariou, M.D., Ph.D., Jan Bělohlávek, M.D., Ph.D., Jan Hovdenes, M.D., Ph.D., Manoj Saxena, M.B., B.Ch., Ph.D., Hans Kirkegaard, M.D., D.M.Sc., Paul J. Young, M.D., Ph.D., Paolo Pelosi, M.D., Christian Storm, M.D., Ph.D., Fabio S. Taccone, M.D., Ph.D., Michael Joannidis, M.D., Clifton Callaway, M.D., Ph.D., Glenn M. Eastwood, R.N., Ph.D., Matt P.G. Morgan, M.B., B.Ch., Ph.D., Per Nordberg, M.D., Ph.D., David Erlinge, M.D., Ph.D., Alistair D. Nichol, Ph.D., Michelle S. Chew, M.D., Ph.D., Jacob Hollenberg, M.D., Ph.D., Matthew Thomas, M.B., B.Ch., Jeremy Bewley, M.B., B.Ch., Katie Sweet, R.N., B.Sc., Anders M. Grejs, M.D., Ph.D., Steffen Christensen, M.D., Ph.D., Matthias Haenggi, M.D., Anja Levis, M.D., Andreas Lundin, M.D., Joachim Düring, M.D., Simon Schmidbauer, M.D., Thomas R. Keeble, M.B., B.S., M.D., Grigoris V. Karamasis, M.D., Claudia 
Schrag, M.D., Edith Faessler, M.D., Ondrej Smid, M.D., Ph.D., Michal Otáhal, M.D., Ph.D., Marco Maggiorini, M.D., Pedro D. Wendel Garcia, M.Sc., Paul Jaubert, M.D., Jade M. Cole, R.N., B.Sc., Miroslav Solar, M.D., Ph.D., Ola Borgquist, M.D., Ph.D., Christoph Leithner, M.D., Samia Abed-Maillard, M.Sc., Leanlove Navarra, R.N., B.Sc., Martin Annborn, M.D., Ph.D., Johan Undén, M.D., Ph.D., Iole Brunetti, M.D., Akil Awad, M.D., Peter McGuigan, M.B., B.Ch., Roy Bjørkholt Olsen, M.D., Ph.D., Tiziano Cassina, M.D., Ph.D., Philippe Vignon, M.D., Ph.D., Halvor Langeland, M.D., Theis Lange, Ph.D., Hans Friberg, M.D., Ph.D., and Niklas Nielsen, M.D., Ph.D.

The authors' affiliations are as follows: the Department of Clinical Sciences Lund, Sections of Cardiology (J. Dankiewicz, D.E.), Neurology (T. Cronberg, G.L.), and Anesthesiology and Intensive Care (H. Levin, O.B.), Skåne University Hospital Lund, Lund University and Clinical Studies Sweden — Forum South, Skåne University Hospital (S.U.), Lund; the Department of Clinical Sciences Lund, Section of Anesthesia and Intensive Care, Skåne University Hospital Malmö, Malmö, (J. Düring, S.S., H.F.); the Department of Clinical Sciences Lund, Sections of Anesthesiology and Intensive Care (M.A., N.N.) and Clinical Sciences Helsingborg (N.N.), Helsingborg Hospital, Helsingborg; the Department of Clinical Sciences Lund, Section of Anesthesiology and Intensive Care Lund, Hallands Hospital, Halmstad (J.U.); the Department of Anesthesiology and Intensive Care Medicine, Institute of Clinical Sciences, Sahlgrenska Academy, University of Gothenburg, Gothenburg (C.R., A. Lundin); the Department of Clinical Science and Education, Center for Resuscitation Science, Karolinska Institutet, Södersjukhuset, Stockholm (P.N., J. Hollenberg, A.A.); and the Department of Anesthesiology, Intensive Care, and Acute Medicine, Linköping University, Linköping (M.S.C.) - all in Sweden; Copenhagen Trial Unit, Center for Clinical Intervention Research, Copenhagen University Hospital (J.C.J.), and the Section of Biostatistics, Faculty of Health and Medical Sciences (T.L.), University of Copenhagen, Copenhagen, the Department of Regional Health Research, the Faculty of Health Sciences, University of Southern Denmark, Odense (J.C.J.), the Research Center for Emergency Medicine, the Department of Clinical Medicine (H.K.), and the Department of Intensive Care (A.M.G., S.C.), Aarhus University Hospital, Aarhus — all in Denmark; Adult Critical Care, University Hospital of Wales, Cardiff (M.P.W., M.P.G.M., J.M.C.), the Department of Intensive Care, Bristol Royal Infirmary, Bristol (M.T., J. Bewley, K.S.), Essex Cardiothoracic Centre, Basildon (T.R.K., G.V.K.), Anglia Ruskin University School of Medicine, Chelmsford, Essex (T.R.K., G.V.K.), and the Department of Anesthesiology and Intensive Care, Royal Victoria Hospital, Belfast (P.M.) - all in the United Kingdom; Neuroscience Critical Care Research Group and Adult Intensive Care Medicine Service, Centre Hospitalier Universitaire Vaudois-Lausanne University Hospital and University of Lausanne, Lausanne (M. Oddo, S.A.-M.), the Departments of Intensive Care Medicine (M.H.) and Anesthesiology and Pain Medicine, Inselspital (A. Levis), Bern University Hospital, University of Bern, Bern, the Intensive Care Department, Kantonsspital St. Gallen, St. Gallen (C. Schrag, E.F.), the Institute of Intensive Care Medicine, University Hospital Zurich, Zurich (M.M., P.D.W.G.), and the Cardiac Anesthesia and Intensive Care Department, Instituto Cardiocentro Ticino, Lugano (T. Cassina) - all in Switzerland; Descartes University of Paris and Cochin University Hospital, Paris (A.C., P.J.), Medical-Surgical Intensive Care Unit, Dupuytren Teaching Hospital, Limoges (P.V.) — all in France; the 2nd Department of Medicine J. Bělohlávek, O.S.), and the Department of Anesthesiology and Intensive Care Medicine (M. Otáhal), General University Hospital and First Faculty of Medicine, Charles University, Prague, the 1st Department of Internal Medicine-Cardioangiology, University Hospital Hradec Králové, and Faculty of Medicine, Charles University, Hradec Králové (M. Solar) - all in the Czech Republic; the Department of Anesthesiology, Division of Emergencies and Critical Care, Oslo University Hospital, Rikshospitalet, Oslo (J. Hovdenes), the Department of Anesthesiology, Sprlandet Hospital, Arendal (R.B.O.), the Department of Anesthesiology and Intensive Care Medicine, St. Olav's University Hospital, and the Department of Circulation and Medical Imaging, Faculty of Medicine and Health Sciences, Norwegian University of Science and Technology, Trondheim (H. Langeland) - all in Norway; the Division of Critical Care and Trauma, George Institute for Global Health, and Bankstown-Lidcombe Hospital, South Western Sydney Local Health District, Sydney (M. Saxena), and the Australian and New Zealand Intensive Care Research Centre, School of Public Health and Preventive Medicine (G.M.E., A.D.N.), and the Department of Intensive Care, Alfred Health (A.D.N.), Monash University, Melbourne - all in Australia; the Medical Research Institute of New Zealand, Intensive Care Unit, Wellington Hospital, Wellington (P.J.Y., L.N.); the Departments of Surgical Sciences and Integrated Diagnostics (P.P.) and Anesthesiology and Intensive Care, San Martino Policlinico Hospital, IRCCS for Oncology and Neuroscience (P.P., I.B.), University of Genoa, Genoa, Italy; the Department of Nephrology and Medical Intensive Care (C. Storm), and Klinik und Hochschulambulanz für Neurologie (C.L.), Charité Universitätzmedizin, Berlin, Germany; the Department of Intensive Care, Erasme University Hospital, Université Libre de Bruxelles, Brussels (F.S.T.); the Division of Intensive Care and Emergency Medicine, Department of Internal Medicine, Medical University Innsbruck, Innsbruck, Austria (M.J.); the Department of Emergency Medicine, University of Pittsburgh, Pittsburgh (C.C.); and University College Dublin Clinical Research Centre at St. Vincent's University Hospital, Dublin, Ireland (A.D.N.),

\section{REFERENCES}

1. Nolan JP, Soar J, Cariou A, et al. European Resuscitation Council and European Society of Intensive Care Medicine guidelines for post-resuscitation care 2015: section 5 of the European Resuscitation Council guidelines for resuscitation 2015. Resuscitation 2015;95:202-22.

2. Panchal AR, Bartos JA, Cabañas JG, et al. Part 3: adult basic and advanced life support: 2020 American Heart Association guidelines for cardiopulmonary resuscitation and emergency cardiovascular care. Circulation 2020;142(16_suppl_2): S366-S468.

3. Hypothermia after Cardiac Arrest Study Group. Mild therapeutic hypothermia to improve the neurologic outcome after cardiac arrest. N Engl J Med 2002; 346:549-56.
4. Bernard SA, Gray TW, Buist MD, et al. Treatment of comatose survivors of outof-hospital cardiac arrest with induced hypothermia. N Engl J Med 2002;346:55763.

5. Lascarrou J-B, Merdji H, Le Gouge A, et al. Targeted temperature management for cardiac arrest with nonshockable rhythm. N Engl J Med 2019;381:2327-37. 6. Nielsen N, Wetterslev J, Cronberg T, et al. Targeted temperature management at $33^{\circ} \mathrm{C}$ versus $36^{\circ} \mathrm{C}$ after cardiac arrest. N Engl J Med 2013;369:2197-206.

7. Kirkegaard H, Sфreide E, de Haas I, et al. Targeted temperature management for 48 vs 24 hours and neurologic outcome after out-of-hospital cardiac arrest: a randomized clinical trial. JAMA 2017; 318:341-50.
8. Nielsen N, Friberg H, Gluud C, Herlitz J, Wetterslev J. Hypothermia after cardiac arrest should be further evaluated a systematic review of randomised trials with meta-analysis and trial sequential analysis. Int J Cardiol 2011;151:333-41.

9. Zeiner A, Holzer M, Sterz F, et al. Hyperthermia after cardiac arrest is associated with an unfavorable neurologic outcome. Arch Intern Med 2001;161:2007-12. 10. Dankiewicz J, Cronberg T, Lilja G, et al. Targeted hypothermia versus targeted normothermia after out-of-hospital cardiac arrest (TTM2): a randomized clinical trialrationale and design. Am Heart J 2019; 217:23-31.

11. Jakobsen JC, Dankiewicz J, Lange T, et al. Targeted hypothermia versus targeted normothermia after out-of-hospital car- 
diac arrest: a statistical analysis plan. Trials 2020;21:831.

12. Iyer VN, Mandrekar JN, Danielson RD, Zubkov AY, Elmer JL, Wijdicks EFM. Validity of the FOUR score coma scale in the medical intensive care unit. Mayo Clin Proc 2009;84:694-701.

13. Jacobs I, Nadkarni V, Bahr J, et al. Cardiac arrest and cardiopulmonary resuscitation outcome reports: update and simplification of the Utstein templates for resuscitation registries: a statement for healthcare professionals from a task force of the International Liaison Committee on Resuscitation (American Heart Association, European Resuscitation Council, Australian Resuscitation Council, New Zealand Resuscitation Council, Heart and Stroke Foundation of Canada, InterAmerican Heart Foundation, Resuscitation Councils of Southern Africa). Circulation 2004;110:3385-97.

14. G $\phi$ tzsche PC. Blinding during data analysis and writing of manuscripts. Control Clin Trials 1996;17:285-290, discussion 290-293.

15. Haywood K, Whitehead L, Nadkarni VM, et al. COSCA (Core Outcome Set for Cardiac Arrest) in adults: an advisory statement from the International Liaison Committee on Resuscitation. Circulation 2018;137(22):e783-e801.

16. van Swieten JC, Koudstaal PJ, Visser MC, Schouten HJ, van Gijn J. Interobserver agreement for the assessment of handicap in stroke patients. Stroke $1988 ; 19$; 604-7.

17. Lilja G, Nielsen N, Ullén S, et al. Protocol for outcome reporting and followup in the Targeted Hypothermia versus Targeted Normothermia after Out-ofHospital Cardiac Arrest trial (TTM2). Resuscitation 2020;150:104-12.

18. Jakobsen JC, Gluud C, Wetterslev J, Winkel P. When and how should multiple imputation be used for handling missing data in randomised clinical trials a practical guide with flowcharts. BMC Med Res Methodol 2017;17:162.

19. R Core Team. R: a language and environment for statistical computing. Vienna: Foundation for Statistical Computing, 2013.

20. Cronberg T, Greer DM, Lilja G, Moulaert V, Swindell P, Rossetti AO. Brain injury after cardiac arrest: from prognostication of comatose patients to rehabilitation. Lancet Neurol 2020;19:611-22.
21. Patterson T, Perkins GD, Hassan Y, et al. Temporal trends in identification, management, and clinical outcomes after out-of-hospital cardiac arrest: insights from the Myocardial Ischaemia National Audit Project database. Circ Cardiovasc Interv 2018;11(6):e005346.

22. Sterne JAC, Savović J, Page MJ, et al. RoB 2: a revised tool for assessing risk of bias in randomised trials. BMJ 2019;366: 14898.

23. Button KS, Ioannidis JPA, Mokrysz C, et al. Power failure: why small sample size undermines the reliability of neuroscience. Nat Rev Neurosci 2013;14:365-76.

24. Pereira TV, Horwitz RI, Ioannidis JPA. Empirical evaluation of very large treatment effects of medical interventions. JAMA 2012;308:1676-84.

25. Olai $\mathrm{H}$, Thornéus $\mathrm{G}$, Watson $\mathrm{H}$, et al. Meta-analysis of targeted temperature management in animal models of cardiac arrest. Intensive Care Med Exp 2020; 8:3.

26. Kelly FE, Nolan JP. The effects of mild induced hypothermia on the myocardium: a systematic review. Anaesthesia 2010;65: 505-15.

Copyright $\odot 2021$ Massachusetts Medical Society.

RECEIVE IMMEDIATE NOTIFICATION WHEN AN ARTICLE IS PUBLISHED ONLINE FIRST

To be notified by email when Journal articles are published online first, sign up at NEJM.org. 